\title{
Knowledge and misconceptions of Saudi women about sexually transmitted infections
}

\author{
Original \\ Article \\ Amal Balbeesi and Saad Mohizea \\ Department of Dermatology, King Khalid University Hospital, King Saud University, Riyadh, \\ Saudi Arabia
}

\begin{abstract}
Background: Sexually transmitted infections (STIs) are increasingly becoming one of the most important health challenges, especially among women, as they bear long-term consequences such as infertility and cervical cancer owing to STIs. This study aimed to assess the knowledge and misconceptions of Saudi women about STIs.

Participants and Methods: A descriptive study was conducted among adult women attending the dermatology clinic at King Saud University Medical City, Riyadh, Saudi Arabia, for reasons other than STIs. They were randomly approached and asked to complete an anonymous prevalidated questionnaire. Uneducated females were guided by an interviewer during the filling up of the survey questionnaire. Of 1150 women approached, 843 adult Saudi women responded to the survey (response rate: $73.3 \%$ ).

Results: The mean age was $30.6 \pm 10.9$ years. Knowledge of STIs was highest for HIV $(85.9 \%)$ and lowest for genital warts $(17.0 \%)$. Respondents believed that genital pruritus, foul discharge, and painful micturition are symptoms of STIs (71.1, 69.1, and 56.4\%, respectively). A considerable percentage believed that STIs are transmitted by masturbation (54.8\%), sleeping on contaminated beds (39.3\%), and by eating and drinking from contaminated utensils $(26.3 \%)$ whereas $8 \%$ believed that STIs are transmitted by shaking hands. Nearly two-thirds of women thought that condoms protect against STIs. Knowledge on the modes of transmission and symptoms of STIs was significantly correlated with younger age, higher level of education, and higher monthly income.

Conclusion and recommendations: Despite the huge campaigns and information about STIs seen on the internet and television by these women, there is still scarcity of knowledge on the more important aspects of STIs, especially the recognition of clinical features and modes of transmission/spread of STIs. There is still a need for health authorities to intensify information-dissemination campaigns about STIs to the grassroots level, particularly among women in Saudi Arabia.
\end{abstract}

Received: 09 April 2017, Accepted: 30 January 2018

Key Words: knowledge, misconceptions, Saudi Arabia, sexually transmitted infections, women.

Corresponding Author: Amal Balbeesi, MD, Department of Dermatology, King Khalid University Hospital, King Saud University, Riya dh, Saudi Arabia Tel.: +966 150521 9873, P.O. Box 12474. Fax : +966-114545651 E-mail: saad660@hotmail.com

Journal of the Egyptian Public Health Association, ISSN: 0013-2446, Vol. 92, No.4

\section{INTRODUCTION}

Sexually transmitted infections (STIs) remain among the most important health challenges in most parts of the world. The estimated global annual incidence of curable STIs in 2012 excluding HIV and viral hepatitis among women aged $15-49$ years was $4.2 \%$ for chlamydia, $0.8 \%$ for gonorrhea, $5.0 \%$ for trichomoniasis, and $0.5 \%$ for syphilis [1]. A study in Saudi Arabia (2006) showed that among the 39049 cases of reported STIs, the common causes included nongonococcal urethritis (14 557 cases), trichomoniasis (10 967 cases), gonococcal urethritis (5547 cases), syphilis (3385 cases), genital warts (1382 cases), genital herpes (216 cases), and Chancroid (78 cases) [2]. The emergence and spread of HIV and AIDS have made the management and control of STIs much more difficult because of the immunosuppression brought about by HIVtreatment drugs, particularly in areas where HIV is very prevalent.
One of the hallmarks of preventive medicine is information and dissemination campaigns. In 2009, the WHO highlighted the importance of a 'comprehensive STI control strategy' that includes targeted community-based interventions, promotion and provision of prevention, and effective clinical services as well as reliable data to guide the response [3]. Furthermore, the WHO emphasizes the value of information and referrals to clinical services, as these promote a decline in incidence and prevalence of STIs in the long run.

Studies on school and university students showed high knowledge of STIs and their predisposing factors but widespread misinformation and misconceptions about contraceptives [4-6]. Studies comparing knowledge across time indicated that a higher level of knowledge was associated with a more risky behavior, and that there is still a large number of people who perceive that they are not at risk for infection particularly HIV/AIDS among 
youngsters [7,8]. Knowledge of STIs and HIV was high (91.8 and $74.04 \%$, respectively) in a study conducted among Indian female students [9]. General awareness of common STIs were also high up to $95.5 \%$ (for gonorrhea) among antenatal patients in Nigeria [10]. We conducted this study to determine the knowledge and misconceptions of Saudi women attending the dermatology clinic at King Saud University Medical City, King Saud University, Riyadh, Saudi Arabia, on STIs.

\section{PARTICIPANTS AND METHODS}

This cross-sectional descriptive study was carried out at King Saud University Medical City, King Saud University in Riyadh, Saudi Arabia and was approved by the Institutional Review Board of the College of Medicine, King Saud University. A convenient sample of adult Saudi women attending the dermatology clinic for reasons other than STIs between January and December 2015 were approached and asked to complete a prevalidated survey questionnaire. The questionnaire was tested by running a pilot test of questionnaire on five women on two different times. The Cronbach's $\alpha$ was 0.87 , indicating a good internal consistency of the questionnaire.

The questionnaire included the following data: sociodemographic characteristics, knowledge concerning STIs such as herpes, genital warts, syphilis, HIV (including symptoms, source and mode of transmission, and preventive measures), source of knowledge, and personal or family history of STI. Knowledge was assessed by three main questions (knowledge on transmission, symptoms, and complications), and 12 questions for misconceptions and beliefs. The responses to the questions were answered by ticking on the boxes allocated for 'yes' or 'no'. Uneducated women were guided by an interviewer during the filling out of the survey questionnaire.

Strict confidentiality was maintained by keeping the questionnaires anonymous. We distributed around 1150 survey questionnaires, and 843 filled-up survey questionnaires were completed (response rate: $73.3 \%$ ).

\section{Statistical analysis}

Data were analyzed using the Statistical Package for Social Sciences, version 23 (SPSS Inc., IBM, Chicago, Illinois, USA). Results are presented in numbers and percentages. Correlations of categorical variables were done using the Pearson correlation test. $\mathrm{P}$ values of less than 0.05 were considered statistically significant.

\section{RESULTS}

The mean age (SD) for all 843 respondents was $30.6 \pm 10.9$ years (range: $18-67$ years). Table 1 shows the demographic characteristics of the respondents.

Table 1.Demographic characteristics of the sample of adult Saud women attending the dermatology clinic, King Saud University Medical City, Riyadh, Saudi Arabia (2015)

\begin{tabular}{lc}
\hline Demographic variables & $\mathrm{n}(\%)$ \\
\hline Age groups (years) & $515(61.1)$ \\
$\leq 30$ & $148(17.6)$ \\
$31-39$ & $126(14.9)$ \\
$40-49$ & $54(6.4)$ \\
$\geq 50$ & \\
Marital status & $387(45.9)$ \\
$\quad$ Single & $371(44.0)$ \\
$\quad$ Married & $85(10.1)$ \\
$\quad$ Divorced/widow & \\
Educational status & $456(54.1)$ \\
College/Postgraduate degree & $341(40.4)$ \\
High school & $46(5.5)$ \\
Elementary & \\
Monthly income & $362(42.9)$ \\
$\geq 5000$ Saudi riyals/month & $481(57.1)$ \\
$<5000$ Saudi riyals per month &
\end{tabular}

Table 2 shows the knowledge of these women on the different aspects of STIs. More than half of the women knew that gonorrhea, syphilis, and HIV/AIDS are STIs (66.0, 57.0, and $85.9 \%$, respectively). Knowledge of HIV/AIDS as an STI was the highest (85.9\%) among all other diseases. However, only $37.2 \%$ of women knew that Herpes is a STI. Approximately $70 \%(70.9 \%)$ of women thought that they can still have STI when engaging in sexual activities during menstruation. A similar percentage $(71.1 \%)$ of the women knew that an itchy perineum (genital pruritus) is a symptom of STI, and also with painful/ difficulty in micturition (56.4\%). Approximately $69.1 \%$ of the women thought that STIs produce vaginal discharges, whereas $58.9 \%$ thought that STIs can bring about genital ulcers, and $35.2 \%$ thought that STIs produce enlarged lymph nodes and ulcers. There were 578 (64.5\%) women who agreed that condoms can give them protection against STIs. Similarly, $61.8 \%$ of women thought that vaccines prevent STIs. 
Table 2. Knowledge about STIs among Saudi women attending the dermatology clinic at King Saud University Medical City, Riyadh, Saudi Arabia (2015)

\begin{tabular}{lc}
\hline Knowledge variables & \% of correct response \\
\hline Knowledge on the sexual transmission of STIs & \\
Gonorrhea is sexually transmitted & 66.0 \\
Syphilis is sexually transmitted & 57.0 \\
Herpes is sexually transmitted & 37.2 \\
HIV is sexually transmitted & 85.9 \\
Genital wart is sexually transmitted & 17.0 \\
STIs can be transmitted through sexual & 70.9 \\
activity during menstruation & \\
Knowledge on the symptoms of STIs & 58.9 \\
Genital ulcers & 71.1 \\
Genital pruritus & 56.4 \\
Difficulty in urination & 69.1 \\
Genital foul discharge & \\
Knowledge on the complications of STIs & 69.5 \\
Cervical cancer & 60.9 \\
Infertility & 61.3 \\
Congenital fetal anomalies &
\end{tabular}

STIs, sexually transmitted infections.

Table 3 shows the beliefs and misconceptions of these women on STIs. There were $9.8 \%$ of the women who thought that tuberculosis is sexually transmitted. Similarly, misconceptions that some diseases are STIs include smallpox (1.9\%), eczema (9.9\%), and genital dryness (54.5\%). Moreover, $54.8 \%$ of women thought that STIs are transmitted by masturbation, 39.3\% through sleeping on contaminated beds, $26.3 \%$ through eating and drinking from contaminated utensils, and $8.0 \%$ through shaking of hands. Furthermore, $61.8 \%$ of women thought that taking oral contraceptives protects them from having STIs, and $32.8 \%$ thought that eating vegetables also prevents them from contracting an STI.

Table 3. Misconceptions and beliefs of Saudi women attending the Dermatology clinic at King Saud University Medical City, Riyadh, Saudi Arabia (2015)

\begin{tabular}{lc}
\hline Misconceptions and beliefs & \% Agree \\
\hline Tuberculosis is sexually transmitted & 9.8 \\
Smallpox is sexually transmitted & 37.2 \\
Eczema is sexually transmitted & 9.9 \\
STIs produce large lymph nodes and ulcers & 35.2 \\
STIs can be transmitted by shaking of hands & 8.0 \\
STIs can be transmitted by masturbation & 54.8 \\
STIs can be transmitted through foods and drinks & 26.3 \\
STIs can be transmitted through contaminated beds & 39.3 \\
Genital dryness is a complication of STIs & 54.5 \\
Gangrene is a complication of STIs & 21.5 \\
Taking oral contraceptive protects one from STIs & 61.8 \\
Eating vegetables prevents one from contracting STIs & 32.8 \\
\hline
\end{tabular}

STIs, sexually transmitted infections.
The knowledge on the modes of transmission and symptoms of STIs was significantly correlated with younger age, higher level of educations, and those with higher monthly income. Respondents who had university and above university levels of education had a higher knowledge level than respondents who have elementary and high school level of education $(\mathrm{r}=0.365, \mathrm{P}<0.001)$. Respondents who have a monthly income of 5000 Saudi riyals and above had higher knowledge level of STIs than those who have a monthly income of less than 5000 SR a month $(\mathrm{r}=0.219, \mathrm{P}<0.001)$. Younger women have better knowledge of STIs compared with older women $(\mathrm{r}=-0.118$, $\mathrm{P}=0.001$ ) (Table 4). Logistic regression analysis showed that the higher level of education is the most significant correlate of better overall knowledge about STIs $(\mathrm{P}<0.001)$.

Table 4. Correlates of knowledge of STIs among adult Saudi women attending the dermatology clinic at King Saud University Medical City, Riyadh, Saudi Arabia (2015)

\begin{tabular}{lcc}
\hline Variables & Correlation coefficient & P value \\
\hline Educational level & & \\
$\quad$ Above university level & 0.365 & $<0.001$ \\
$\quad$ Elementary and high school level & \\
Monthly income & & \\
$\quad$ 5000 SR and above & 0.219 & $<0.001$ \\
$\quad$ Less than 5000 SR a month & & \\
Age & -0.118 & 0.001 \\
\hline
\end{tabular}

\section{DISCUSSION}

This study showed that only 5-6 of 10 Saudi women who attended the Dermatology Clinic of King Saud University Medical City in Riyadh, Saudi Arabia, in 2015 knew about STIs such as gonorrhea and syphilis, whereas 8-9 of 10 Saudi women knew well what HIV/AIDS is. This may be owing to the fact that other STIs such as syphilis, genital warts, and gonorrhea have been overshadowed by the extensive HIV/AIDS campaigns on the mass media (TV and internet). Our result on the knowledge of HIV/AIDS is higher compared with the study conducted among Indian female students in 2005 [9]. However, our results on the knowledge of other STIs particularly gonorrhea are lower compared with the study conducted among female patients attending the antenatal clinic in a Nigerian hospital in 2001 [10]. The discrepancies in the rates between our studies and the Indian and Nigerian studies may be attributed to the age of the population. Our study surveyed a wide range of age from 18 to 67 years old, whereas the Indian study focused on female students 16-25 years old, and the Nigerian study focused on the antenatal (married) females.

There is still 5-6 of 10 Saudi women who thought that STIs can be transmitted through masturbation, and 2-3 of 10 Saudi women thought that eating and drinking from contaminated utensils may expose them to have STIs. There seems to be some discrepancies between knowledge (what 
they know) of STIs and their belief and misconceptions on how STIs are transmitted, meaning they know that such STI exists but have poor knowledge on how it is transmitted and much more how the disease progresses.

This study reaffirms that misinformation and misconceptions about STIs do exist as pointed out by Chacko et al. [5]. Despite the advancement of technology where television programs and the internet can widely inform the general public about STIs, still a large proportion of the population, particularly the mothers and their children, is not aware of STIs or is misinformed by relatives or friends about STIs. It is very important to note that more than half of our respondents are educated women, and more than $90 \%$ have at least secondary education; however, there is a lower percentage of women who were knowledgeable enough to know how these STIs are transmitted and how they will respond to such disease. This is probably because of the absence of the right of Saudi women to openly discuss sexual health without being controlled by the rules of the society, culture, and tradition as pointed out by Mwambete and Mtaturu [6]. Discussing such issues in a society that deems such disease unacceptable especially among women served as a roadblock toward proper information and even unsolicited consultations to health authorities.

This is the first study to our knowledge that surveyed adult Saudi women and tackled the issue of STIs. The leading daily newspaper Arab News published an article (Al-Jassem) related to lack of awareness on STIs in October 2010. The article stated that 'The reason for this misunderstanding is due to lack of information on these diseases and lack of sexual health awareness among couples.' Moreover, it stated that 'there are many reasons for this lack of awareness on STIs among Saudis, one of them is due to the obstacles Saudi society places on discussing STIs, which are backed by social and ethical reasons, and that 'people in Saudi Arabia cannot discuss STDs because they believe such issues are taboos in the society,' [11]. Furthermore, in that article by Al-Jassem, 92.0\% of surveyed Saudis thought that education about STIs should be taught in schools, and not only through mass media, where most of the respondents gain their information on STIs. Despite these however, the levels of knowledge and misconceptions of Saudis, the women in particular, have not been thoroughly addressed, and hence the prevalence of STIs continue to rise. Ignorance about STIs increases a woman's risk for serious health consequences such as infertility and cervical cancer. Ample knowledge of STIs can help detect the disease early and when combined with proper treatment and follow-up is an effective tool to protect one's health.

\section{Study limitations}

One probable limitation of our study is the homogeneity of our study population. More than $90 \%$ of our respondents are well educated, in fact more than half has a college or postgraduate degree; therefore, drawing a solid conclusion on the relationship between level of education and knowledge of STIs may not be that decisive. Another reason is the convenient sampling method wherein we invited all Saudi women who came for consultation aside from STIs to the dermatology clinic. We recommend further larger surveys on Saudi women on their knowledge of STIs particularly in the rural areas, or those in the community setting.

\section{CONCLUSION AND RECOMMENDATIONS}

Knowledge of STIs especially its transmission and complications is still low among adult Saudi women despite the huge campaigns and information dissemination conducted by the Saudi health authorities. There is still a need for health authorities to intensify their campaign and reach out further particularly to the community level. There is a need to introduce and incorporate sexual education as part of health education in institutions that is well supported not only by the health authorities and practitioners but by the society itself.

\section{Conflicts of interest}

The authors have no affiliations with or involvement in any organization or entity with any financial interest

\section{REFERENCES}

1. Newman L, Rowley J, Vander Hoom S, Wjesooriya NS, Unemo M, Low N, et al. Global estimates of the prevalence and incidence of curable sexually transmitted infections in 2012 based on systematic review and global reporting. PLoS ONE 2015; 10:e0143304.

2. Madani TA. Sexually transmitted infections in Saudi Arabia. BMC Infect Dis 2006; 6:3.

3. Steen R, Wi TE, Kamali A, Ndowa F. Control of sexually transmitted infections and prevention of HIV transmission: mending a fractured paradigm. Bull World Health Org 2009; 87:858-865.

4. Sekirime WK, Tamale J, Lule JC, Wabwire-Mangen F. Knowledge, attitude and practice about sexually transmitted diseases among university students in Kampala. Afr Health Sci 2001; 1:16-22.

5. Chacko S, Kipp W, Laing L, Kabagambe G. Knowledge of and perceptions about sexually transmitted diseases and pregnancy: a qualitative study among adolescent students in Uganda. J Health Pop Nutr 2007; 25:319-327.

6. Mwambete KD, Mtaturu Z. Knowledge of sexually transmitted diseases among secondary school students in Dar el Salaam, Tanzania. Afr Health Sci 2006; 6:165-169.

7. Milsom I, Andersson-Ellstrom A. Knowledge about the prevention of sexually transmitted disease: a longitudinal study of young women from 16-23 years of age. Sex Trans Infect 2002; 78: 339-341. 
8. Ferreira MP, Grupo de Estudos em Populacao, Sexualidade e Aids. Knowledge and risk perception of the Brazilian population on HIV/Aids, 1998 and 2005. Rev Public Health 2008; 42(Suppl 1):65-71.

9. Hazarika NC, Mahanta J. Perceptions of unmarried young women regarding family size, sexually transmitted diseases at residential regional institutes in northeastern India. Southeast Asian J Trop Med Public Health 2005; 35:233-236.
10. Obiechina NJ, Nwosu OB, Okafor CI, Ikpeze OC. Knowledge, awareness and perception of sexually transmitted diseases (STDs) among antenatal patients at Nnewi, Nigeria. J Obstet Gynaecol 2001; 21:292-294.

11. Al-Jassem D. Saudis lack awareness on STIs. Riyadh: Arab News, Saudi Arabia, 2010, Wed, 2010-10-27 22:37 [cited 2017 March 3]. Available from: http://www.arabnews.com/node/358883 